\title{
Pirólise catalítica do PEBD usando como catalisador a vermiculita modificada
}

\section{Catalytic pyrolysis of LDPE using modified vermiculite as a catalyst}

\author{
Franciel Aureliano Bezerra ${ }^{1}$, Aneliése Lunguinho Figueiredo², Antonio Souza de Araujo² e \\ Ana Paula de Melo Alves Guedes*
}

\author{
'Universidade Federal de Uberlândia - UFU, Uberlândia, MG, Brasil \\ 2Universidade Federal do Rio Grande do Norte - UFRN, Natal, RN, Brasil \\ *anachemistry@hotmail.com
}

\begin{abstract}
Resumo
O polietileno de baixa densidade (PEBD) é um dos polímeros mais usados atualmente, e a grande quantidade desse polímero produzida resulta em toneladas de resíduos, que necessitam ser tratados. Neste trabalho foi realizada a pirólise termocatalítica do PEBD usando como catalisador a argila vermiculita modificada, como alternativa para o tratamento dos resíduos. A argila foi tratada com solução de ácido nítrico a diferentes concentrações e calcinada a $400{ }^{\circ} \mathrm{C}$. Os materiais foram caracterizados por técnicas de difratometria de raios X, termogravimetria, adsorção de nitrogênio e espectroscopia de energia dispersiva. A pirólise térmica e termocatalítica foi realizada em um micro reator acoplado com $\mathrm{GC} / \mathrm{MS}$, a $500{ }^{\circ} \mathrm{C}$. O intuito da pirólise de resíduos poliméricos é a obtenção de hidrocarbonetos leves $(\mathrm{C}<16)$, que possam ser empregados na indústria química e petroquímica, através de quebras na cadeia polimérica. Os resultados foram satisfatórios, com aumento no rendimento para hidrocarbonetos leves ao empregar os catalisadores chegando a $71,4 \%$ de produtos com $\mathrm{C}<16$, enquanto a pirólise térmica resultou apenas de $25,8 \%$.
\end{abstract}

Palavras-chave: vermiculita, argila, pirólise, PEBD.

\begin{abstract}
Low density polyethylene (LDPE) is one of the most commonly-used polymers currently, and the great quantity of this polymer produced results in tons of waste that must be treated. We studied the thermocatalytic pyrolysis of LDPE with a modified clay vermiculite catalyst as an alternative for treatment of waste. The clay was treated with a solution of nitric acid at different concentrations and calcined at $400^{\circ} \mathrm{C}$. The materials were characterized by X-ray diffraction, thermogravimetry, nitrogen adsorption, and energy dispersive spectroscopy. Thermal and thermocatalytic pyrolysis were carried out in a microreactor coupled with $\mathrm{GC} / \mathrm{MS}$ at $500{ }^{\circ} \mathrm{C}$. The aim of the polymeric waste pyrolysis is the obtainment of light hydrocarbons $(\mathrm{C}<16)$, which can be used in the chemical and petrochemical industry, through breaks in the polymer chain. The results were satisfactory, with an increase in yield for light hydrocarbons by using catalysts reaching up to $71.4 \%$ of products with $\mathrm{C}<16$, whereas thermal pyrolysis resulted in only $25.8 \%$.
\end{abstract}

Keywords: vermiculite, clay, pyrolysis, LDPE.

\section{Introdução}

O polietileno de baixa densidade (PEBD) é um polímero parcialmente cristalino (50-60\%), cuja temperatura de fusão está na região de 110 a $115^{\circ} \mathrm{C}$, com uma combinação única de propriedades: tenacidade, alta resistência ao impacto, alta flexibilidade, boa processabilidade, estabilidade e propriedades elétricas notáveis ${ }^{[1]}$. Devido essas propriedades o PEBD é uma das resinas termoplásticas mais consumidas no Brasil. Segundo a ABIPLAST ${ }^{[2]}$ seu consumo foi cerca de 14,5\% do total de plásticos consumidos no Brasil em 2011.

Devido ao rápido crescimento da demanda de produtos plásticos, impulsionado pela diversidade de propriedades e aplicações, nos últimos anos as atenções estão voltadas para os

resíduos plásticos gerados pós-consumo, visto que a maioria dos plásticos não são biodegradáveis e na maioria dos casos têm um baixo tempo de uso, contribuindo significativamente para o problema da gestão de resíduos urbanos.

Para evitar a poluição ambiental, os resíduos plásticos em particular o PEBD devem ser reciclados e recuperados. Muitos estudos têm sido realizados quanto à reciclagem terciária de resíduos plásticos através de pirólise térmica e termocatalítica. Esse processo é considerado uma alternativa promissora para a conversão de resíduos plásticos em produtos químicos de baixo peso molecular, que podem ser 
utilizados como matérias-primas para a indústria química e petroquímica.

De acordo com a literatura, o uso de catalisadores na pirólise apresenta algumas vantagens em relação a processos térmicos, como menor consumo de energia, tempo de reação mais curto e uma boa seletividade para produtos de maior valor comercial ${ }^{[3]}$. Vários tipos de catalisadores utilizados na pirólise de poliolefinas têm sido estudados: ZSM- $5^{[4]}$, H-ZSM-5 ${ }^{[5]}$, MCM-41 ${ }^{[6]}$, H-AL-MCM-41 ${ }^{[7]}$, SAPO ${ }^{[8,9]}$, como também trabalhos que utilizam argilas, como Lama vermelha $^{[10]}$ e Caulim ${ }^{[3]}$.

O baixo custo, alta resistência térmica, além da estrutura que possibilita a criação de poros e aumento da área superficial após tratamentos, como calcinação e lixiviação ácida, tornam as argilas materiais alternativos para a catálise ${ }^{[11]}$. A lixiviação ácida promove o aumento de acidez, necessária para o uso em pirólise; o controle das condições de tratamento ácido (concentração do ácido, temperatura, tempo, razão ácido/argila) é fator determinante para as propriedades, tais como acidez, área superficial, porosidade e atividade catalítica, dos materiais resultantes ${ }^{[12-16]}$. De acordo com Stefanis $^{[11]}$, as argilas atuam na pirólise promovendo a quebra das ligações poliméricas, através dos seus sítios ácidos superficiais de Brönsted e Lewis.

A argila vermiculita possui propriedades importantes para catalisadores como alta resistência térmica e grande potencial para formação de sítios ácidos do tipo Brönsted, devido ao seu alto número de substituições tetraédricas ${ }^{[17-20]}$. O presente trabalho teve como objetivo avaliar a seletividade dos materiais obtidos por lixiviação seletiva da argila vermiculita frente à pirólise termocatalítica do $\mathrm{PEBD}$, sendo os produtos principais hidrocarbonetos leves de maior valor de mercado.

\section{Materiais e Métodos}

\subsection{Lixiviação ácida da argila vermiculita}

Utilizou-se a argila vermiculita proveniente da região de Santa Luzia (PB), Brasil. Inicialmente, fez-se uma limpeza na argila com água destilada, e depois à mesma adicionou-se $10 \mathrm{~mL}$ de solução de $\mathrm{HNO}_{3}$ a 2, 3 e 4 mol.L $\mathrm{L}^{-1}$ por grama de argila e, manteve-se sob agitação a $90^{\circ} \mathrm{C}$ por $4 \mathrm{~h}$. Em seguida, a mistura foi decantada e o sólido obtido foi lavado com água destilada. $\mathrm{O}$ sólido foi filtrado e seco a $80^{\circ} \mathrm{C}$ por $24 \mathrm{~h}$. A nomenclatura utilizada para os sólidos obtidos foi $\mathrm{V} 0$ para vermiculita de partida e Vx para vermiculita tratada com ácido, sendo x referente à concentração ácida utilizada no tratamento. Realizou-se a calcinação dos materiais a $400{ }^{\circ} \mathrm{C}$, com taxa de aquecimento de $10^{\circ} \mathrm{C} \cdot \mathrm{min}^{-1}$, por $4 \mathrm{~h}$, em atmosfera ambiente.

\subsection{Caracterizações}

As análises de difração de raios X (DRX) foram realizadas em um equipamento modelo Mini Flex II da RIGAKU, utilizando radiações de $\mathrm{CuK} \alpha$, com abertura da fenda de $0,15^{\circ}$, passo de $0,02^{\circ}$, na faixa $2 \theta$ entre $2-70^{\circ}$. Analisou-se os resultados utilizando o programa PCPDFWIN 2003. As análises termogravimétrica foram realizadas em um equipamento modelo Q600 TG/DSC da TA Instruments, usando, aproximadamente, $8 \mathrm{mg}$ de amostra, aquecimento de 30 à $900{ }^{\circ} \mathrm{C}$, taxa de $10{ }^{\circ} \mathrm{C} \cdot \mathrm{min}^{-1}$, fluxo de $\mathrm{N} 2$ de $50 \mathrm{~mL} \cdot \mathrm{min}^{-1}$, em um cadinho de alumina de $90 \mu \mathrm{L}$. Para as análises de adsorção/dessorção de nitrogênio foi utilizado um equipamento modelo Nova 1200e, da QuantaChrome Instruments; o material foi pré-tratado a $300^{\circ} \mathrm{C}$ por $3 \mathrm{~h}$ sob vácuo e a adsorção de nitrogênio foi realizada $\mathrm{a}-196{ }^{\circ} \mathrm{C}$, com o uso de nitrogênio líquido, numa faixa de pressão parcial entre 0,01-0,95. A lixiviação seletiva das amostras foi acompanhada por espectroscopia de energia dispersiva (EDS), em um microscópio eletrônico de varredura modelo 1430, da marca Leo, equipado com espectrômetro de energia dispersiva.

\subsection{Determinação da acidez}

O procedimento foi realizado utilizando cerca de $100 \mathrm{mg}$ dos materiais. As amostras foram adicionadas em um sistema cotendo um forno, com controle da temperatura, onde foram aquecidas até $400{ }^{\circ} \mathrm{C}$ por $2 \mathrm{~h}$ sob fluxo de $\mathrm{N}_{2}\left(100 \mathrm{~mL} \cdot \mathrm{min}^{-1}\right)$; em seguida, a temperatura foi reduzida a $95^{\circ} \mathrm{C}$, e as amostras foram deixadas sob vapor de n-butilamina, conduzido por fluxo de $\mathrm{N}_{2}$ por $1 \mathrm{~h}$ (fluxo de nitrogênio controlado, de modo que a n-butilamina fosse borbulhada); depois, o fluxo de n-butilamina foi cessado e as amostras ficaram $1 \mathrm{~h}$ sob fluxo de $\mathrm{N}_{2}\left(100 \mathrm{~mL} \cdot \mathrm{min}^{-1}\right)$, sendo, em seguida, esfriadas até a temperatura ambiente. Cerca de $8 \mathrm{mg}$ dos materiais obtidos foram analisados em um equipamento Q600 TG/DSC da TA Instruments, em cadinho de alumina de $90 \mu \mathrm{L}$, sendo aquecidos até $900{ }^{\circ} \mathrm{C}$, taxa de $10^{\circ} \mathrm{C} \cdot \mathrm{min}^{-1}$ e fluxo de $\mathrm{N}_{2}$ de $50 \mathrm{~mL} \cdot \mathrm{min}^{-1}$. Tal técnica permite, através da interação da amina com o material, determinar a força dos seus sítios ácidos, baseando-se na temperatura em que ocorrem as perdas de massa, visto que quanto mais elevada a temperatura da perda, maior é a interação da amina com o sítio ácido, e consequentemente, maior é a acidez do material ${ }^{[13,16,21]}$.

\subsection{Processo de pirólise do PEBD}

A pirólise térmica e catalítica do PEBD foi realizada em um pirolisador modelo PY-2020iS Control da Frontier LAB, acoplado a um GC/MS modelo QP 2010 da Shimadzu. Foi utilizado um cadinho de aço inoxidável, de $50 \mu \mathrm{L}$, em atmosfera de gás hélio de $3 \mathrm{~mL} \cdot \mathrm{min}^{-1}$, a $500{ }^{\circ} \mathrm{C}$. A identificação dos produtos da pirólise foi feita com o banco de dados da biblioteca NIST (National Institute of Standards of Technology) do software acoplado ao sistema de análise GC/MS

\section{Resultados e Discussão}

\subsection{Caracterização dos catalisadores}

Através dos resultados de DRX, observou-se que a vermiculita perde sua cristalinidade gradualmente, à medida que a concentração ácida empregada foi sendo elevada, tanto que os materiais V3 e V4 apresentam caráter essencialmente amorfo, ver Figura 1. Essa amorfização dos materiais é decorrente da destruição da estrutura da argila pela lixiviação dos metais do esqueleto inorgânico. Para as amostras V0 e V2 observa-se um deslocamento da reflexão $001^{(*)}$ para valores maiores de $2 \theta$, reflexão característica do empilhamento das lamelas da argila, sendo o deslocamento atribuído à diminuição do espaçamento basal causada pela 


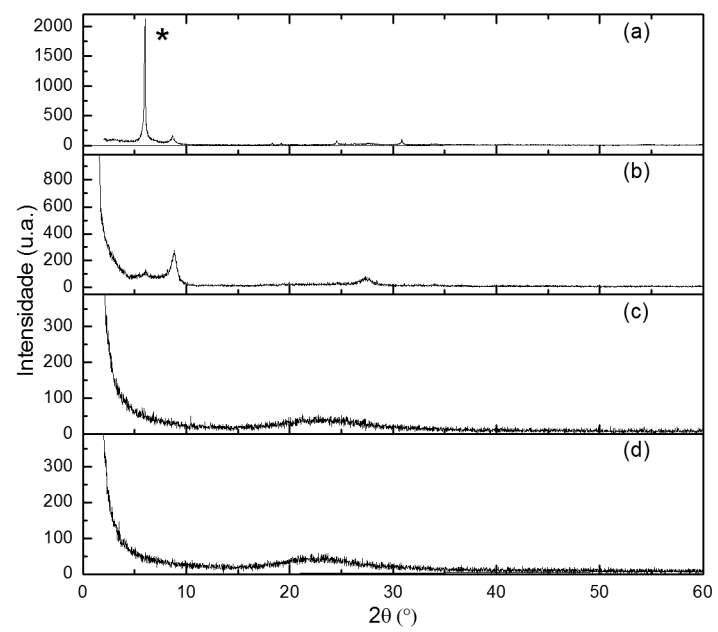

Figura 1. Difratogramas de raios $X$ das amostras V0(a), V2(b), V3(c) e V4(d).

saída de água durante a calcinação. A lixiviação dos metais constituintes do esqueleto inorgânico da argila foi confirmada pela análise química apresentada na Tabela 1 .

Na Tabela 1 observa-se que a lixiviação é acompanhada pelo o aumento da área específica $\left(\mathrm{S}_{\mathrm{BET}}\right)$ até o sólido V3, entretanto, no tratamento a 4 mol.L $\mathrm{L}^{-1}$ (V4), houve um decréscimo da valor de $\mathrm{S}_{\mathrm{BET}}$, que é atribuído a fusão dos microporos formando mesoporos, diminuindo assim a área específica; tem-se também uma diminuição nos valores de acidez média com o tratamento ácido, o que é esperado pela retirada dos sítios de $\mathrm{Al}^{3+} \mathrm{e} \mathrm{Fe}^{3+}$, acidez de Lewis ${ }^{[22]}$, e o aumento nos valores de acidez fraca é concordante com o aumento percentual de $\mathrm{SiO}_{2}$ dos materiais, e conseqüente aumento dos grupos silanóis $(\mathrm{SiOH})$, principais responsáveis pela acidez fraca ${ }^{[23]}$; outra fonte de sítios de Brönsted são os prótons $\left(\mathrm{H}^{+}\right)$, que substituem os cátions interlamelares das argilas, devido a lixiviação ${ }^{[24]}$.

\subsection{Pirólise catalítica do PEBD}

$\mathrm{O}$ cromatograma da pirólise térmica realizada com o PEBD é apresentado na Figura 2, destacando-se a maior obtenção em produtos de cadeia carbônica longas, ou seja, hidrocarbonetos pesados. O resultado da pirólise termocatalítica do PEBD é apresentado na Figura 3, onde se percebe claramente que produtos mais leves são favorecidos pela presença dos catalisadores V0 e V2, enquanto os materiais V3 e V4 não foram satisfatórios para a conversão.

Através da quantificação dos picos presentes nos cromatogramas listada na Tabela 2, o sólido V2 apresentou uma seletividade $10 \%$ maior para a formação de produtos leves com cadeias com menos de dezesseis carbonos $(\mathrm{C}<16)$ em relação ao sólido V0 e esse comportamento é atribuído à maior área específica deste sólido, mais de 20 vezes superior, que significa maior área disponível para a ocorrência de quebras das ligações das cadeias poliméricas; no entanto, o mesmo não é observado para o sólido V3, que possui aproximadamente o dobro da área específica do sólido V2. Esses resultados indicam que a seletividade dos materiais se deve a uma relação de idealidade entre as

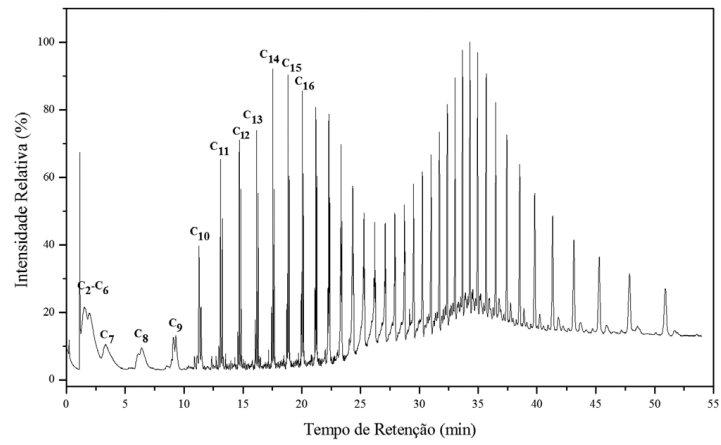

Figura 2. Cromatograma da pirólise térmica do PEBD.

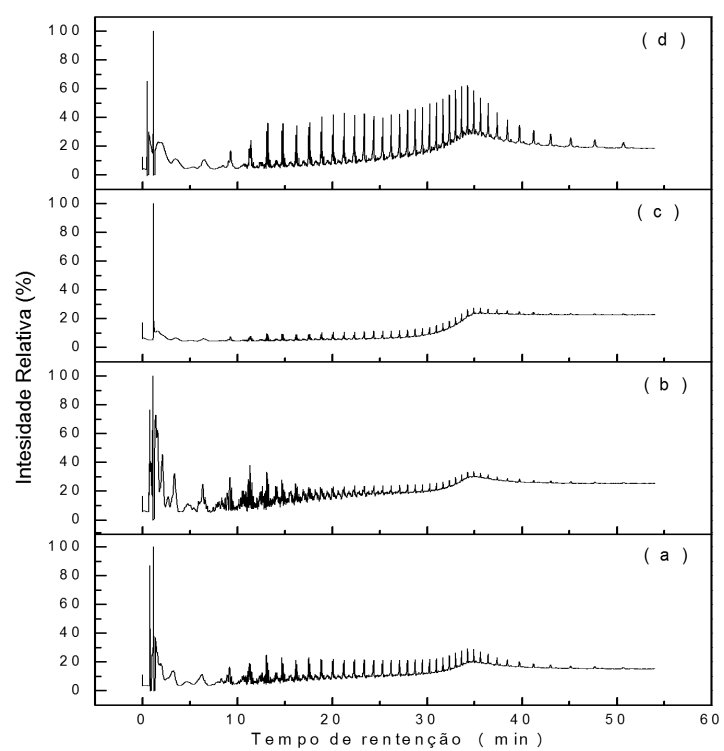

Figura 3. Cromatograma da pirólise termocatalítica do PEBD com os catalisadores V0 (a), V2(b), V3(c) e V4(d).

Tabela 1. Características das amostras de vermiculita, antes e após o tratamento ácido.

\begin{tabular}{ccccccc}
\hline & & \multicolumn{2}{c}{$\begin{array}{c}\text { Acidez } \\
\text { Amostras }\end{array}$} & $\begin{array}{c}\mathbf{S}_{\text {BET }} \\
\left(\mathbf{m}^{2} \mathbf{g}^{-1}\right)\end{array}$ & \multicolumn{2}{c}{$\begin{array}{c}\text { Composição Química } \\
\text { Pmol.g }\end{array}$} \\
\cline { 3 - 7 } & & Fraca & Média & $\mathbf{S i O}_{2}$ & $\mathbf{A l}_{2} \mathbf{O}_{3}$ & $\mathbf{F e}_{2} \mathbf{O}_{3}$ \\
\hline V0 & 16 & 0 & 0.264 & 44.62 & 9.18 & 5.46 \\
V2 & 339 & 0.680 & 0.220 & 55.80 & 5.01 & 4.04 \\
V3 & 673 & 1.050 & 0.230 & 58.80 & 0.88 & 1.37 \\
V4 & 575 & 0.580 & 0.190 & 61.30 & 0.62 & 0.79 \\
\hline
\end{tabular}

Tabela 2. Faixas de distribuição dos produtos da reação de pirólise térmica e termocatalítica do PEBD.

\begin{tabular}{ccrccc}
\hline \multirow{2}{*}{ Pirólise } & \multicolumn{5}{c}{ Produto (\%) } \\
\cline { 2 - 6 } & $\boldsymbol{\Sigma}_{\text {C2-C16 }}$ & C2-C4 & C5-C10 & C11-C16 & C16> \\
\hline PEBD & 25.8 & 1.7 & 13.0 & 11.1 & 74.2 \\
PEBD+V0 & 62.2 & 5.5 & 42.0 & 14.7 & 37.8 \\
PEBD+V2 & 71.4 & 20.0 & 41.2 & 10.2 & 28.6 \\
PEBD+V3 & 30.7 & 8.5 & 15.0 & 7.2 & 69.3 \\
PEBD+V4 & 28.0 & 1.2 & 15.7 & 11.1 & 72.0 \\
\hline
\end{tabular}


propriedades texturais, com as propriedades químicas da superfície da argila.

A aplicação de uma concentração muito elevada de ácido diminuiu muito o percentual de alumínio de V3 e V4, sendo uma desvantagem para a atividade catalítica na pirólise do PEBD, mesmo havendo um pronunciado aumento na área específica. Segundo Okada et al. ${ }^{12}$, há uma relação ideal entre o percentual de alumínio, responsável pela acidez de Brönsted, e as condições da lixiviação ácida realizada, que é usada para promover um aumento na área específica. Entretanto, essa relação é altamente dependente do material de partida, visto que a constituição da argila é dependente do seu local de origem.

Através da distribuição dos produtos da pirólise por faixas de número de carbono na cadeia, Tabela 2, observa-se que o catalisador V2 promove uma maior conversão em produtos na faixa de carbonos de $\mathrm{C} 2-\mathrm{C} 16$, que compreende o GLP (C2-C4), gasolina (C5-C10) e destilados médios (C11-C16), evidenciando assim a maior atividade catalítica desse material, entre todos os estudados. Ao comparar os resultados de V2 e V0, observa-se que o V0 teve uma maior seletividade na faixa da gasolina e dos destilados médios, porém sua conversão total na faixa $\mathrm{C} 2-\mathrm{C} 16$ foi inferior à de V2, comportamento atribuído ao maior percentual de alumínio em sua estrutura, visto que à medida que íons $\mathrm{Al}^{3+}$ são lixiviados, há aumento da acidez de Lewis, responsável por quebras das ligações C-C e C-H do $\mathrm{PEBD}^{[22]}$. Em relação à pirólise do $\mathrm{PEBD}$ na presença dos catalisadores V3 e V4, os sólidos não apresentam atividade catalítica satisfatória para obtenção de hidrocarbonetos leves, pois ao comparar os produtos da pirólise térmica, não há alteração significativa no percentual de produtos leves formados.

A fim de verificar o efeito de um material intermediário entre V0 e V2, a pirólise foi realizada com o material V1, Figura 4. Como pode ser visto, o material possibilitou o aumento de produtos leves, em relação à pirólise sem catalisador, porém, foi menos eficiente que V2. Os percentuais de faixas de átomos de carbono $(\mathrm{C} 2-\mathrm{C} 4=16,8 ; \mathrm{C} 5-\mathrm{C} 10=35,5$; $\mathrm{C} 11-\mathrm{C} 16=8,0$ e $\mathrm{C}>16=39,7)$, mostraram que V2 foi mais eficiente nas três faixas de produtos leves, indicando a necessidade de uma relação ideal entre área superficial e percentual de alumínio, apresentada por V2.

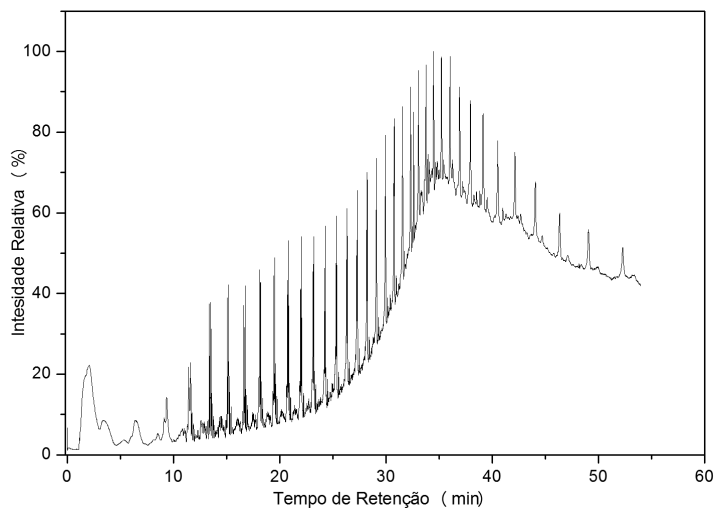

Figura 4. Cromatograma da pirólise termocatalítica do PEBD com o catalisador V1.
Um diferencial que torna o material V2 mais eficiente frente ao $\mathrm{V} 0$ é em respeito à formação de produtos na faixa de C2-C4. O gás liquefeito de petróleo (GLP) é de grande importância para toda a população mundial, do ponto de vista econômico e por ter a vantagem de ser uma fonte de energia limpa. Essa grande importância se traduz no consumo, no Brasil, de cerca de $10 \mathrm{mil} \mathrm{m}^{3}$ no ano de $2011^{[25]}$.

\section{Conclusões}

A lixiviação ácida causou a diminuição dos percentuais de metais presentes na estrutura da vermiculita, originando sílicas porosas, com grande aumento de área específica. A utilização de concentrações ácidas muito elevadas causa a destruição da estrutura dos materiais, resultando em sólidos amorfos, e diminui drasticamente o percentual de metais presentes, diminuindo a atividade catalítica. Os resultados demonstram que a atividade dos catalisadores e a distribuição dos produtos são dependentes do grau de lixiviação que a argila é submetida. O material V2 resultou na maior conversão em produtos leves, tendo então a maior eficiência catalítica para a pirólise do PEBD. Por outro lado, V3 e V4 não tiveram atividade catalítica satisfatória, pois as conversões obtidas foram próximas aos resultados da pirólise do PEBD ausente de catalisador. Além disso, o material obtido com concentração ácida de 1 mol.L-1 $(\mathrm{V} 1)$ foi menos eficiente que V2, indicando ser o que apresenta melhor relação área superficial e conteúdo de alumínio. Em suma, o uso de catalisadores oriundos da argila vermiculita na pirólise termocatalítica é uma opção atraente para a reciclagem do PEBD, tendo em vista a obtenção de percentuais significativos de hidrocarbonetos leves, que podem ser utilizados pela indústria química e petroquímica, sendo este catalisador um material de baixo custo.

\section{Agradecimentos}

A Capes, ao CNPq e ao PRH-PB 22 pelo o apoio a pesquisa.

\section{Referências}

1. Coutinho, F. M. B., Mello, I. L., \& Santa Maria, L. C. (2003). Polietileno: Principais tipos, propriedades e aplicações. Polímeros. Ciência e Tecnologia, 13(1), 1-13. http://dx.doi. org/10.1590/S0104-14282003000100005.

2. Associação Brasileira da Indústria do Plástico-ABIPLAST. (2011). Perfil 2011: indústria brasileira de transformação de material plástico. São Paulo. Recuperado em 5 de maio de 2014, de http://file.abiplast.org.br/download/estatistica/ perfil2012_versao_eletronica.pdf

3. Panda, A. K., \& Singh, R. K. (2011). Catalytic performances of kaoline and silica alumina in the thermal degradation of polypropylene. Journal of Fuel Chemistry and Technology, 39(3), 198-202. http://dx.doi.org/10.1016/S1872-5813(11)60017-0.

4. López, A., Marco, I., Caballero, B. M., Laresgoiti, M. F., Adrados, A., \& Aranzabal, A. (2011). Catalytic pyrolysis of plastic wastes with two different types of catalysts: ZSM-5 zeolite and Red Mud. Applied Catalysis B: Environmental, 104(3-4), 211-219. http://dx.doi.org/10.1016/j.apcatb.2011.03.030.

5. Coelho, A., Costa, L., Marques, M. M., Fonseca, I. M., Lemos, M. A. N. D. A., \& Lemos, F. (2012). The effect of ZSM-5 zeolite acidity on the catalytic degradation of high- 
density polyethylene using simultaneous DSC/TG analysis. Applied Catalysis A, General, 413-414, 183-191. http://dx.doi. org/10.1016/j.apcata.2011.11.010.

6. Jin, S., Cui, K., Guan, H., Yang, M., Liu, L., \& Lan, C. (2012). Preparation of mesoporous MCM-41 from natural sepiolite and its catalytic activity of cracking waste polystyrene plastics. Applied Clay Science, 56, 1-6. http://dx.doi.org/10.1016/j. clay.2011.11.012.

7. Araújo, A. S., Fernandes, V. J. Jr, Araujo, S. A., \& Ionashiro, M. (2002). Kinetic evaluation of the pyrolysis of high density polyethylene over H-AlMCM-41 material. Studies in Surface Science and Catalysis, 141, 473-478. http://dx.doi.org/10.1016/ S0167-2991(02)80578-X.

8. Singhal, R., Shinghal, C., \& Upadhyayula, S. (2010). Thermalcatalytic degradation of polyethylene over silicoaluminophosphate molecular sieves - A thermogravimetric study. Journal of Analytical and Applied Pyrolysis, 89(2), 313-317. http://dx.doi. org/10.1016/j.jaap.2010.09.007.

9. Fernandes, G. J. T., Fernandes, V. J. Jr, \& Araújo, A. S. (2002). Catalytic degradation of polyethylene over SAPO-37 molecular sieve. Catalysis Today, 75(1-4), 233-238. http:// dx.doi.org/10.1016/S0920-5861(02)00074-3.

10. Adrados, A., Marco, I., Caballero, B. M., López, A., Laresgoiti, M. F., \& Torres, A. (2012). Pyrolysis of plastic packaging waste: A comparation of plastic residuals from material recovery facilities with simulated plastic waste. Waste Management, 32(5), 826-832. http://dx.doi.org/10.1016/j.wasman.2011.06.016. PMid:21795037.

11. Stefanis, A., Cafarelli, P., Gallese, F., Borsella, E., Nana, A., \& Perez, G. (2013). Catalytic pyrolysis of polyethylene: A comparation between pillared and restructured clays. Journal of Analytical and Applied Pyrolysis, 104, 479-484. http://dx.doi. org/10.1016/j.jaap.2013.05.023.

12. Okada, K., Arimitsu, N., Kameshima, Y., Nakajima, A., \& MacKenzie, K. J. D. (2006). Solid acidity of 2:1 type clay minerals activated by selective leaching. Applied Clay Science, 31(3-4), 185-193. http://dx.doi.org/10.1016/j.clay.2005.10.014.

13. Shimizu, K., Higuchi, T., Takasugi, E., Hatamachi, T., Kodama, T., \& Satsuma, A. (2008). Characterization of Lewis acidity of cation-exchanged montmorillonite K-10 clay as effective heterogeneous catalyst for acetylation of alcohol. Journal of Molecular Catalysis A Chemical, 284(1-2), 89-96. http:// dx.doi.org/10.1016/j.molcata.2008.01.013.

14. Melo, D. M. A., Ruiz, J. A. C., Sobrinho, E. V., Melo, M. A. F., Martinelli, A. E., \& Zinner, L. B. (2003). Determination of relative acid strength of $\mathrm{La}$ /paligorskyte by n-butylamine. Journal of Solid State Chemistry, 171(1-2), 217-220. http:// dx.doi.org/10.1016/S0022-4596(02)00154-8.

15. Gil, A., Vicente, M. A., \& Korili, S. A. (2005). Effect of the $\mathrm{Si} / \mathrm{Al}$ ratio on the structure and surface properties of silica- alumina-pillared clays. Journal of Catalysis, 229(1), 119-126. http://dx.doi.org/10.1016/j.jcat.2004.10.013.

16. Hart, M. P., \& Brown, D. R. (2004). Surface acidities and catalytic activities of acid-activated clays. Journal of Molecular Catalysis A Chemical, 212(1-2), 315-321. http://dx.doi. org/10.1016/j.molcata.2003.11.013.

17. Sasca, V., Avram, L., Verdes, O., \& Popa, A. (2010). The n-butyl amine TPD measurement of Brönsted acidity for solid catalysts by simultaneous TG/DTG-DTA. Applied Surface Science, 256(17), 5533-5538. http://dx.doi.org/10.1016/j. apsusc.2009.12.131.

18. Temuujin, J., Okada, K., \& Mackenzie, K. J. D. (2003). Preparation of porous silica from vermiculite by selective leaching. Applied Clay Science, 22(4), 187-195. http://dx.doi. org/10.1016/S0169-1317(02)00158-8.

19. Campos, A. M., Moreno, S., \& Molina, R. (2008). Relationship between hydrothermal treatment parameters as a strategy to reduce layer charge in vermiculite, and its catalytic behavior. Catalysis Today, 133-135, 351-356. http://dx.doi.org/10.1016/j. cattod.2007.12.110.

20. Perez-Maqueda, L. A., Maqueda, C., Perez-Rodriguez, J. L., Subrt, J., Cerny, Z., \& Balek, V. (2012). Thermal behaviour of ground and unground acid leached vermiculite. Journal of Thermal Analysis and Calorimetry, 107(2), 431-438. http:// dx.doi.org/10.1007/s10973-011-1480-2.

21. Perez-Maqueda, J. L., Maqueda, C., Murafa, N., Subrt, J., Balek, V., Pulisová, P., \& Lancok, A. (2011). Study of ground and unground leached vermiculite II. Thermal behaviour of ground acid-treated vermiculite. Applied Clay Science, 51(3), 274-282. http://dx.doi.org/10.1016/j.clay.2010.11.031.

22. Komadel, P., \& Madejová, J. (2006). Acid activation of clay minerals. In F. Bergaya, B. K. G. Theng \& G. Lagaly (Eds.). Handbook of clay science (Developments in Clay Science, Vol. 1, pp. 263-287).

23. Moreno, E. L., \& Rajagopal, K. (2009). Desafios da acidez na catálise em estado sólido. Quimica Nova, 32(2), 538-542. http://dx.doi.org/10.1590/S0100-40422009000200044.

24. Angaji, M. T., Zinali, A. Z., \& Qazvini, N. T. (2013). Study of Physical, Chemical and Morphological Alterations os Smectite Clay upon Activation and Funcionalization via the Acid treatment. World Journal of Nano Science and Engineering, 3(04), 161-168. http://dx.doi.org/10.4236/wjnse.2013.34019.

25. Agência Nacional do Petróleo, Gás Natural e Biocombustíveis - ANP. (2013). Evolução do mercado de combustiveis e derivados: 2000-2012. Brasília. Recuperado em 5 de maio de 2014, de http://www.anp.gov.br/?dw=64307

Enviado: Maio 05, 2014 Revisado: Abr. 08, 2015 Aceito: Jul. 02, 2015 